\title{
Design and Analysis of a Novel Variable Stiffness Joint for Robot
}

\author{
Xiang Zhang ${ }^{1}$, Twan Capehart ${ }^{2}$ and Carl A. Moore ${ }^{3}$ \\ ${ }^{1}$ Master student, Department of Mechanical Engineering, FAMU/FSU College of Engineering, Tallahassee, FL32310, USA \\ ${ }^{2}$ PhD student, Department of Mechanical Engineering, FAMU/FSU College of Engineering, Tallahassee, FL32310, USA \\ ${ }^{3}$ Associate professor, Department of Mechanical Engineering, FAMU/FSU College of Engineering, Tallahassee, FL32310, USA
}

\begin{abstract}
As people pay more attention to the safety of human-robotic interaction, the flexibility of machine joints is becoming more and more important. To address the needs of future robotic applications, many kinds of variable stiffness mechanisms have been designed by scientists. But most of the structures are complex. By studying and comparing many different mechanism designs of variable stiffness joint, we recognize the need to miniaturization and reduce weight of variable stiffness joints with high frequency operation. To address this, need a continuously Variable Compliant Joint (CVCJ) was designed. The core of the joint is based on the structure of the spherical continuously variable transmission (SCVT) which is the catalyst to change the stiffness continuously and smoothly. In this paper, we present a compact variable stiffness joint structure to meet the volume and weight requirements of the future robotic systems. We show the connection between the joint stiffness coefficient and the structure parameters by making mathematical analysis, modelling and simulation for the system to verify the ability to satisfy the base application requirements of the compliant joint.
\end{abstract}

\section{Introduction}

Within recent decades, robotics has rapidly developed and become more sophisticated. Comfortable actuated prostheses, walking and running robots, industrial robot arms and a series of bionic robots are increasingly used in various robotic fields As a result, the interaction and physical contact between the user and the machine are increased. In traditional robotics, they widely use rigid method to make sure the motion precisely [1]. However, in everyday environments, and in the face of more complex tasks, the traditional rigid robot joints are not as beneficial in meeting the requirements of safety and reliability, because it is prone to collisions that can cause damage to the equipment and even cause injury to the user. Safety is the most important requirement in robots physically interacting with humans. Robots working closely to humans collaborating or even physically interacting with them should be intrinsically safe in order to guarantee safety even in case of any sensor's failure or unexpected interaction or break down [1].

Often to increase safety, the engineering community is investigating compliance from many different directions. In fact, the biological counterpart to the variably stiff joint is the biological muscle that has superior functional performance and a neuromechanical control system that is much more advanced at adapting and tuning its parameters [5]. Biologists have long known that mechanical stiffness and compliance in our bodies is indispensable for navigating our world [2]. According to this theory, MIT (Massachusetts Institute of Technology) proposed incorporating series elasticity as a purposeful element within an actuator (The original Series elastic actuators (SEA)) [3]. This actuator has been successfully used in many different robotics like Cog [4]. But the system stiffness of this actuator is constant; it cannot be modified.

Inspired by the biological musculo-tendinous combination of biceps and triceps in the human arm, many current variable stiffness robots use the agonist-antagonist form of passively elastic structure. For example, in 2005 Shane. A. Migliore et al., created a rotational joint by using this idea [6]. The MACCEPA (mechanically adjustable compliance and controllable equilibrium position actuator) [8] provide by Ham et al., and the nonlinear spring tension (NST) unit [9] designed by Dr. Osada is the similar structure. Later in 2014, He Fuben and Liang Yande in China provide an Mimicking Musculo-tendinous joint [7] which is much simpler than previous products. But the disadvantage is the structure's complexity, which in results in a larger size joint.

Leverage mechanism is another widely used variable stiffness structure. In its implementations, the stiffness of 
the elastic element is constant but the stiffness adjustment depends on the effective motion ratios. There are 4 main basic leverage structures shown in figure 1 [19]. Adjusting the stiffness of the structure by varying the position of the springs, the load point of the force, and the pivot point of the lever. At present, most mainstream variable stiffness actuators are based on this principle. AwAs (actuator with adjustable stiffness) [10] adjusts the stiffness by changing the position of the springs, figure 1(a), Carloni's energyefficient variable stiffness actuator [15] is using the figure 1(b), CompAct-VSA (CompAct-variable stiffness actuator), vsaUT-II, and AwAs-II [11-13] are the structure of figure 1(c). Kim designed another similar variant [16], the pivot point is kept constant while the position of the effective arm is changed as shown in figure 1(d). These structures have some limitation in their use due to their larger sizes and complicated structure design. For each of these designs, the longer the lever length, the more precise the stiffness adjustment; however, a longer lever also increases the size of the structure which may be detrimental in some robotic applications.

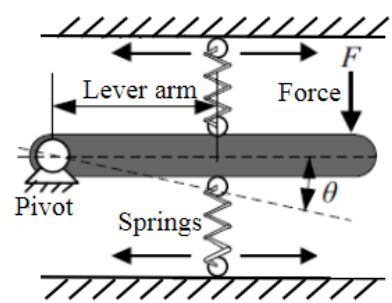

(a)

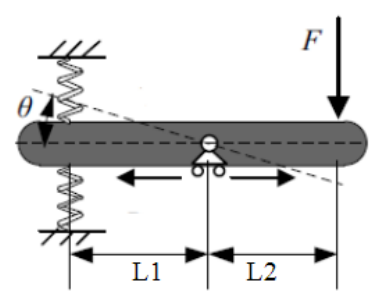

(c)

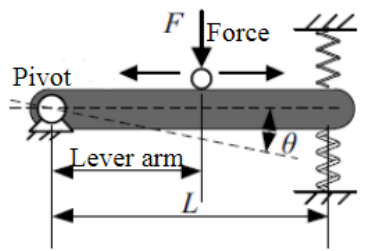

(b)

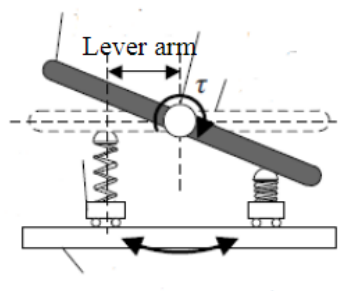

(d)
Figure 1. Schematic of the several adjustable stiffness concepts based on the lever mechanism. (a) Stiffness is adjusted by changing the position of the springs. (b) Stiffness is changed by changing the point of the load. (c) Stiffness is altered by changing the pivot point on the arm. (d) Structure of symmetrical arrangement of springs with respect to the center of rotation.

In this paper we design a novel joint whose small size does not reduce its stiffness precision. Moreover, its smaller size offers a reduction in overall weight. In addition, it can operate at high frequency meaning that we can adjust the stiffness smoothly and quickly.

Therefore, by studying the research of Peshkin and Tadakuma [14], this paper proposes a similar but smaller joint: the CVT-box which is based on the same CVT theories presented in those works. Compared with other variable stiffness joints, it has a less complex design, a lighter weight and smaller size. Through the relevant kinematics analysis and simulation we show that this variable stiffness joint has a stiffness range from zero to infinity in theory. The design provides a practical and effective joint for robot miniaturization and structural simplification.

This paper is organized as follows. This current section served as a review of variable stiffness actuator design. In section 2 we consider the variable stiffness principle and the design of the CVT-box variable stiffness device. Section 3 considers the performance of the CVT-box design through simulation. Finally, in section 4 we conclude and offer areas for future study.

\section{ANALYSIS OF STIFFNESS-ADJUSTING MECHANISM}

\subsection{Concept of the variable stiffness}

According to Hooke's law, the stiffness (k) of a linear spring is fixed:

$$
k=\frac{F}{\Delta x}=\text { constant or } k=\frac{\tau}{\Delta \theta}=\text { constant }
$$

$\mathrm{F}$ is the force exerted by the outside world on the spring, and $\Delta \mathrm{x}$ and $\Delta \theta$ are the translational or rotational changes (respectively) of the initial position of the elastic element. The relationship between force and displacement is linear. However, in a variable stiffness system, the stiffness of the mechanism can vary with position, and the relationship between force and displacement can be nonlinear.

$$
k_{(x)}=\frac{d F}{d x} \neq \text { constant or } k_{(x)}=\frac{d \tau}{d \theta} \neq \text { constant }
$$

There are also some non-linear springs, as shown in the figure 2 , but their adaptability is not widely adopted, and their application is very limited. Most common wire and torsional springs have fixed stiffness; how to use them to design nonlinear and even adjustable stiffness elastic structures is our focus.

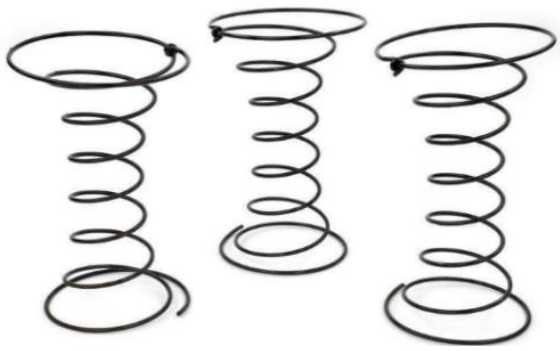

Figure 2. Nonlinear Spring

From studies by C. A. Moore [14-16] and Twan Capehart [2], it was seen that the stiffness of the system can be changed by using a spherical CVT to adjust the speed ratio between the input and output to adjust the deformation of the linear spring.

In the simplest design of the CVT as shown in figure 3 , there are six rollers distributed on the surface of a ball. Each roller is located at the center of each face of circumscribed cube of the ball. All of the rollers are pressed 
into the surface by a preload force by a support structure that is not shown. This preload force keeps each of the roller has a compressed rolling contact with the sphere of the ball and minimizes slip. Of the 6 rollers, there are two called drive rollers which rotate with angle velocities $\omega_{1}$ or $\omega_{2}$. Two other rollers are steering rollers (we only shown one of them, the other is on the underside of the ball and has identical orientation). The steering rollers are free to roll on their axis, but their orientations (steering angle gamma) are identical and controlled by a motor and gearing that is not shown. The last two rollers are followers and help to constrain the sphere.

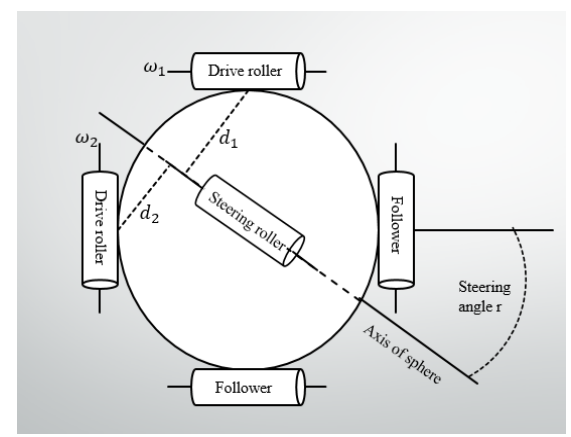

Figure 3. Conceptual model of a continuously variable transmission

The CVT's rolling constraints are such that the angular speed of the drive roller 1 (w1) and drive roller 2 (w2) is a function of the steering roller angle gamma or,

$$
T=\frac{\omega_{2}}{\omega_{1}}=\frac{d_{2}}{d_{1}}=\tan (\gamma)
$$

where $\mathrm{T}$ is the transmission ratio. To create a joint with variable stiffness, we couple the joint's rotation to one of the drive rollers and couple a linear spring to the other drive roller. The detailed design is described in the next section.

\subsection{Design of the flexible joint with variable stiffness}

Figure 4(a) shows the traditional serial elastic actuator SEA model. It can be seen from the figure that the elastic element is connected in series between the driving element and the load. The motor-driven elastic element drives the load to rotate. The total stiffness of the structural system is constant, and its stiffness is also fixed at every position. In comparison to the variable stiffness CVT-box model in Figure 4(b), the elastic elements are connected in series between the CVT-Box and the rack. The load is connected to the Box input shaft, and the elastic element is connected to the output of the Box. By changing the orientation of the steering roller to change the deformation of the elastic element, the system stiffness can be changed to the desired value at any position. We will describe more details in the following paragraphs.

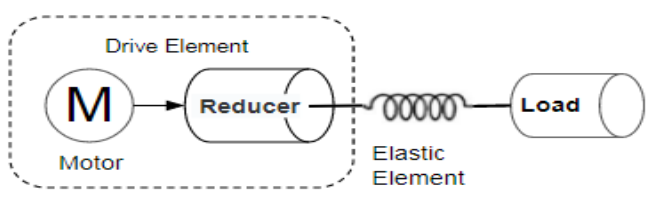

(a)

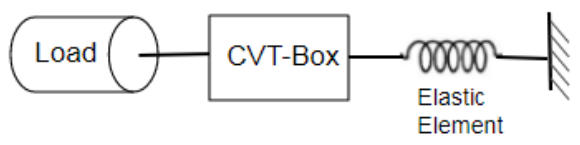

(b)

Figure 4. (a) Diagram of a simplified SEA model. (b) Diagram of a conceptural Variable Stiffness CVT model.

The variable stiffness joint diagram is shown in figure 5. The input drive roller is connected with the input beam. The output drive roller connected with the output lever which is fixed to a spring. When we apply a force at input beam, it will rotate the input drive rooller an angle $\theta_{1}$ then according the theory

$$
T=\frac{\omega_{2}}{\omega_{1}}=\tan (\gamma)
$$

The output drive roller would rotate an angle

$$
\theta_{2}=T * \theta_{1}
$$

which cause deformation of the spring which is the source of the stiffness. By adjusting the transmission ratio $\mathrm{T}$, we can get variable transmission ratio to control the value of the deformation of the spring. As a result we can get different feedback of the input angle with the force application. So we get the variable stiffness. We talk about the details of the stiffness analysis in the following part.

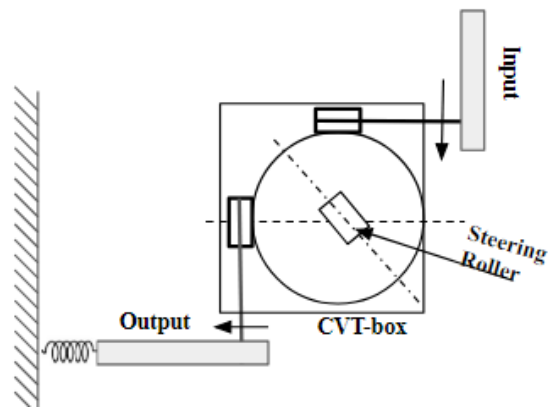

Figure 5. Diagram of the variable stiffness joint set top view.

\subsection{Stiffness Adjustment Analysis}

Now we focus on the force analysis of the joint to verify the concept of the design. The joint stiffness $k(\theta)=$ $d \tau / d \theta$, is defined as the derivative of the elastic torque $\tau$ with respect to the angular deflection $\theta$. As shown in figure 6 , at the beginning position figure 6(a) without any load on it and without any deflection of the spring. Then when we apply a torque on the input shaft, there is angle deflection on both input and output shaft. $\theta_{1}$ is the input 
shaft rotation angle and the $\theta_{2}$ is the output shaft rotation angle which causes deflection of the spring.

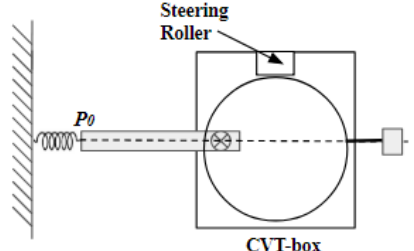

(a) Position 1

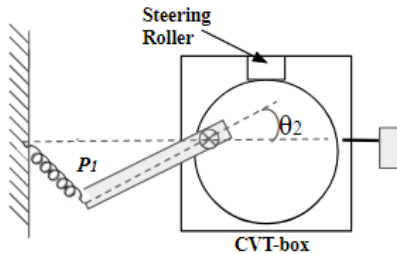

(b) Position 2
Figure 6. Side view of the joint set working process form original position to the final. (a) is the original position of the joint without force and rotation. (b) is position with specifice force load on the input shaft and the output shaft rotate an angle $\theta_{2}$.

First, we develop the mathematic relationship between the deformation of the spring with the output angle. The diagram of the force analysis is shown in figure 7.
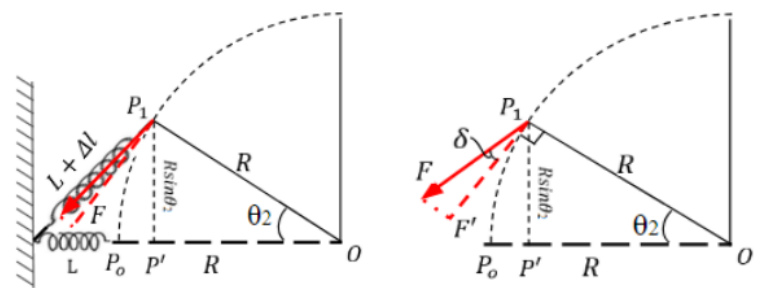

Figure 7. Diagram of Force Analysis. L is the original length of the spring, $\mathrm{R}$ is the length of the output lever which is rotating according to the center $\mathrm{O}$, point $\mathrm{P}$ is the connection point between spring and the lever, $\theta$ is the drive roller rotation angle, $\Delta \mathrm{l}$ is the elongation of the spring, $\delta$ is the angle between the direction of the spring with the perpendicular direction of the radius $\mathrm{R}$.

The elongation of the spring $\Delta l$ can be described by the drive roller rotation angle $\theta_{2}$ as

$$
\Delta l_{\left(\theta_{2}\right)}=\sqrt{R^{2}+L^{2}+2 R L-2 R^{2} \cos \left(\theta_{2}\right)-2 R L \cos \left(\theta_{2}\right)}-L
$$

Then according to the Hooke's law, the spring force $F$ is the product of the elastic modulus and elongation of spring. So, the expression for the force can be written as

$$
F_{\left(\theta_{2}\right)}=k \Delta l
$$

$\delta$ is the angle between the direction of the spring with the perpendicular direction of the radius R. It is easy to get the result by geometric relation,

$$
\delta_{\left(\theta_{2}\right)}=\arctan \frac{R+L-R \cos \left(\theta_{2}\right)}{R \sin \left(\theta_{2}\right)}-\theta_{2}
$$

So the force in the direction of the radius perpendicular is

$$
F_{\left(\theta_{2}\right)}^{\prime}=F_{\left(\theta_{2}\right)} * \cos \left(\delta_{\left(\theta_{2}\right)}\right)
$$

Finally, the torque that is applied to the output drive roller is

$$
\tau_{\left(\theta_{2}\right)}=F_{\left(\theta_{2}\right)}^{\prime} R
$$

From the equation (5), we know the relation between the $\theta_{2}$ and the $\theta_{1}$. So substituting $\theta_{1}$ for $\theta_{2}$ we get,

$$
\begin{aligned}
& \tau_{(t)}=-R k \cos \left[\sin \left(T \theta_{1}\right)+\right. \\
& \left.\frac{\left(L+R-R \cos \left(T \theta_{1}\right)\right) \cos \left(T \theta_{1}\right)}{L R \sin \left(T \theta_{1}\right)-R \sin \left(T \theta_{1}\right) \sqrt{R^{2} \sin ^{2}\left(T \theta_{1}\right)+\left(L+R-R \cos \left(T \theta_{1}\right)\right)^{2}}}\right]
\end{aligned}
$$

The transmission ratio $\mathrm{T}$ and the spring constant $\mathrm{k}$ are the two design parameters. The torque is depend on the elastic constant $\mathrm{k}$ of the spring that are used in the actuator. But since the springs are selected and determined when the joint is designed, the total stiffness is independent with the $\mathrm{k}$. However, in different designs, the rate of the change of the torque can be higher with the $\mathrm{k}$ increase. So in a certain joint, the transmission ratio is the only parameter to be judiciously decided depending on desired torque capacity and deflection range.

Table I shows the parameters used in the model's simulation. The results as shown in figure 8 the output torque increases with larger transmission ratio at same displacement .

Table 1. Mechanical Properties used in the MATLAB Calculation

\begin{tabular}{|c|c|c|}
\hline Parameters & Symbols & Values \\
\hline Output Lever Radius & $\mathrm{R}$ & $0.05 \mathrm{in}$ \\
\hline Spring Original Length & $\mathrm{L}$ & $0.02 \mathrm{in}$ \\
\hline Spring Elastic Modulus & $\mathrm{k}$ & $100 \mathrm{~N} \cdot \mathrm{m}^{-1}$ \\
\hline Roller Diameter & $\mathrm{d}$ & $0.2 \mathrm{in}$ \\
\hline Sphere Diameter & $\mathrm{D}$ & $0.7 \mathrm{in}$ \\
\hline
\end{tabular}

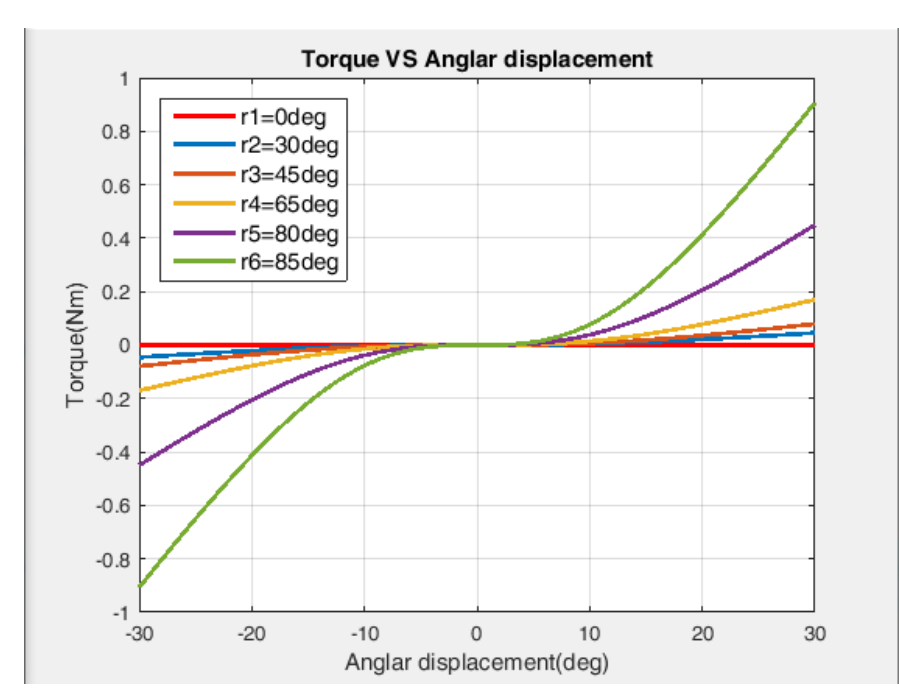

Figure 8. Correlation between the joint angle and the torque output using various CVT transmission angles from $-30^{\circ} \leq$ $\theta_{1} \leq 30^{\circ}$. The curves are symmetric with respect to the 0 angular 
displacement. When $\gamma=0^{\circ}$, the torque are always zero. When $\gamma=90^{\circ}$, the torque will go to infinity.

Then according to the definition of the stiffness, the stiffness of the system is:

$$
K=\frac{d \tau}{d \theta_{1}}=R k * f_{\left(\theta_{1}\right)}
$$

The $f_{\left(\theta_{1}\right)}$ is a function with $\theta_{1}$ as an independent variable. The output stiffness of the joint can be driven by (11) and (12). The result of the stiffness for whole system as show in figure 11 and figure 10 . In figure 9 the displacement is plotted over a range of transmission angle, from 0 to 85 degrees, at 15 degree increments. When the transmission angle approach to 90 degrees, the stiffness would go to infinity, the system would be rigid, theoretically.

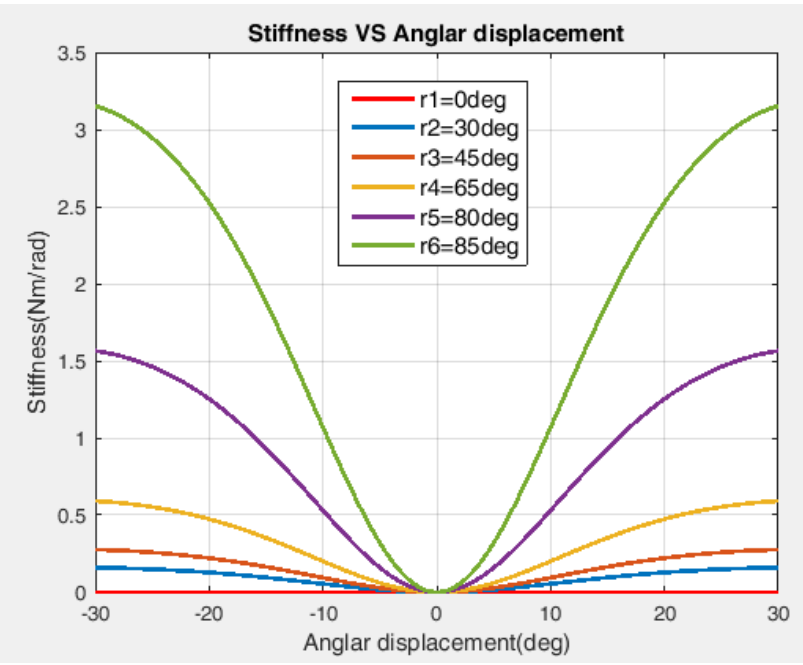

Figure 9. Effective joint stiffness at joint displacements. Changing the CVT-box transmission angle produces different stiffness-displacement curve.

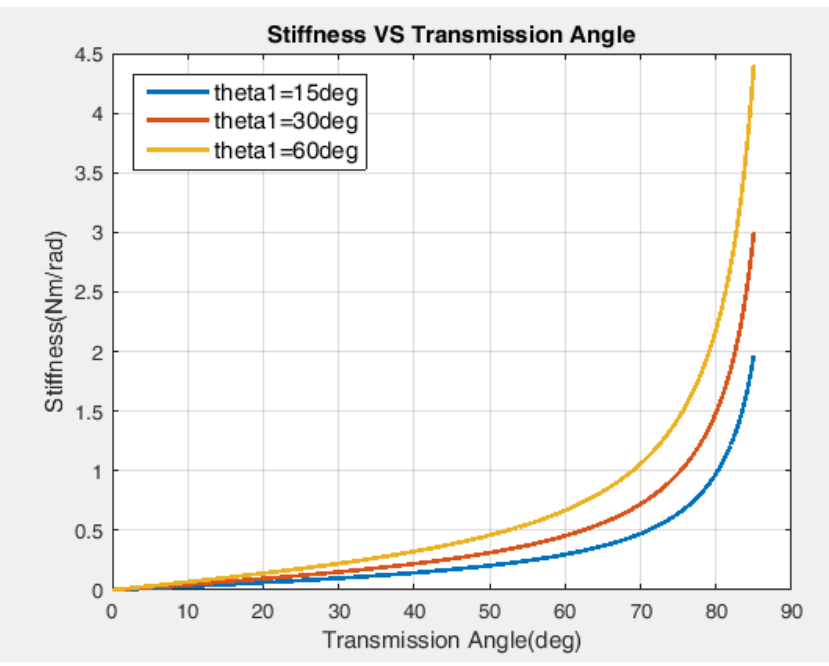

Figure 10. Graphs show the joint stiffness achieved versus various transmission angles at different joint angle displacements.

\section{JOINT PROTOTYPE DESIGN}

The concept of the CVT-Box joint is implemented in variable stiffness joint as it is shown in figure 12 . We show the exploded CAD view of the joint prototype in which the two fundamental actuator mechanisms: the CVT-Box and the elastic elements.

The CVT-Box consists of sphere indicated by 9, the input and output drive rollers indicated by 7 and 3 , and the steering rolles indicated by 5 . The motor will be coupled to the steering roller gear to change the direction of the axis of the sphere to get the expected transmission ratio.

The elastic elements consists of linear extension springs (4), the rotation lever (2), and the fixed pedestal ( 1 ) which is illustrated also in figure 13.

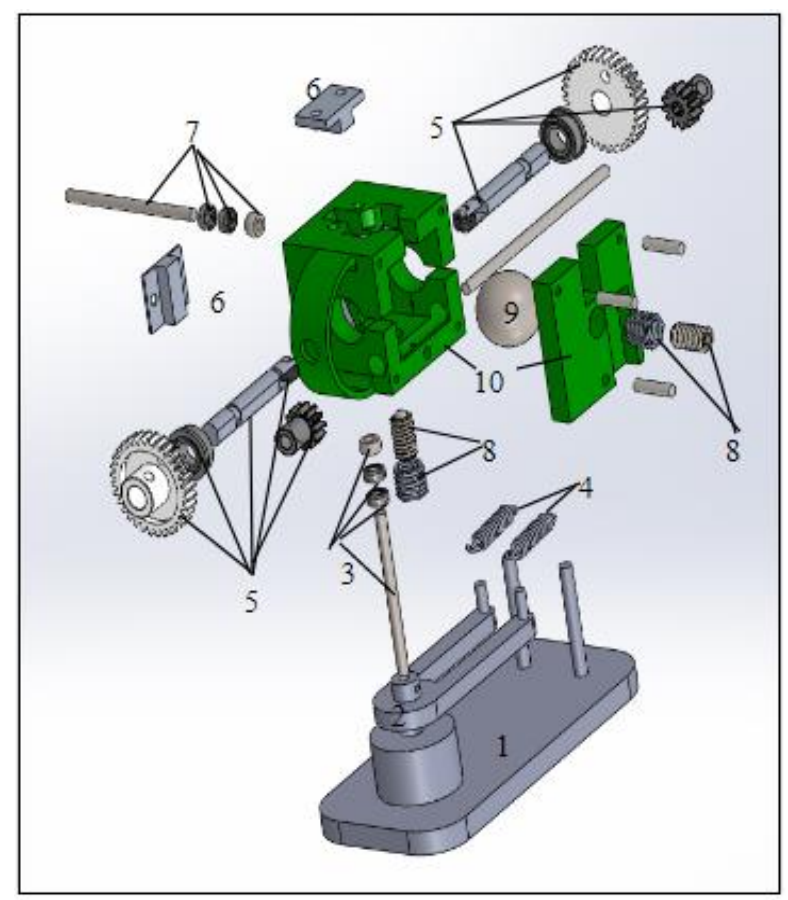

Figure 11. Exploded view of the CVT-Box joint CAD design. The labels is indicate the following: 1 -the fixed reference pedestal ; 2-rotation lever; 3-output shaft with roller and bearings; 4-linear springs; 5-steering shafts with gears, bearings and steering rollers; 6-fix parts; 7-input shaft with roller and bearings; 8-followers; 9-sphere; 10-CVT-Box outside frame housing.

Following from the working principle to describe the mechanism. The fixed reference pedestal is fixed on the ground. The two springs connect the pedestal with the rotation lever which is fixed with output shaft. With the rotation of the output shaft, the springs deform. This is source of the stiffness. The input and output shafts are distributed on the two adjacent sides of the box with 90 degrees. The rollers are fixed on the shaft and close contacted with the sphere by the preload which provided by the followers. The two steering shafts are distributed on two opposite side of the box. There is a roller on each tip of the shaft. The roller are also close connected with the sphere. The gear on the steering shaft is connect with a motor to adjust the transmission angle. There are two fix shaft with followers which are used to provide preload and restrict the sphere to the correct position. So the sphere is 
in rolling contact with all six rollers. Because the center of the sphere is stationary. The sphere's axis of the rotation must pass through the center of the sphere (this can be seen in the figure 3). The four rollers and the two followers must be at the center of each side of the outer tangent cube of the sphere. And the axis of rotation of the sphere must be collinear with the axis of the rotation of the steering roller. All of these parts are restricted by a rigid frame.

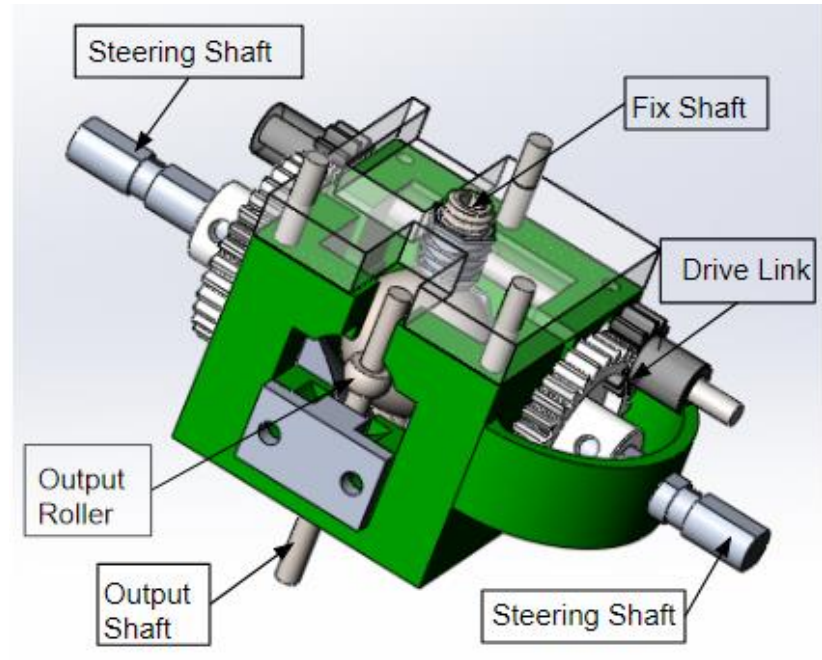

Figure 12. View of the CVT-Box, the core part of the joint used to change the transmission angle.

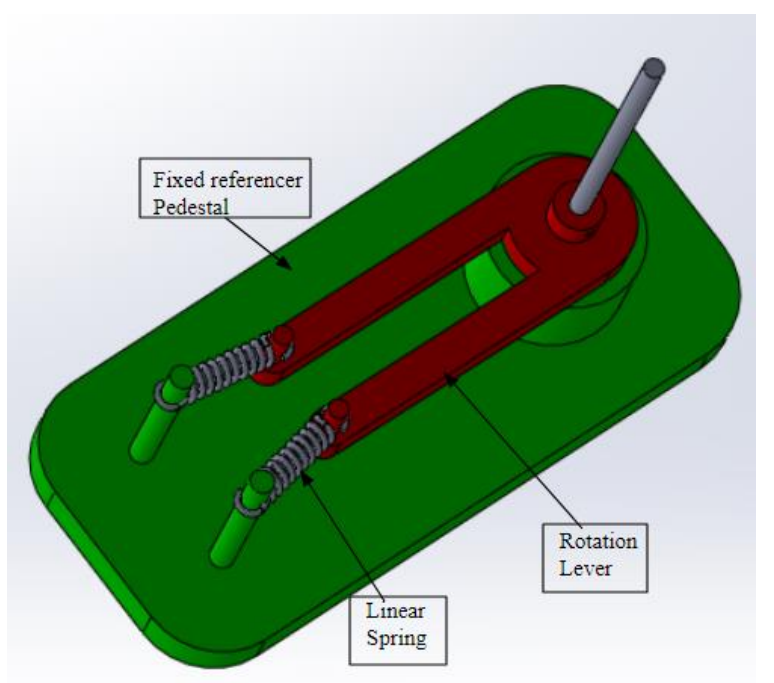

Figure 13. View of the elastic elements.

With this joint designed, we use the $3 \mathrm{D}$ printer to make the outside frame to make sure it has lowest weigh. There is no any force load on the frame directly, so we do not worry about the strength of the frame. Other parts we will build them with aluminium.

Table 2. General specifications

\begin{tabular}{|c|c|c|}
\hline Name & Value & Unit \\
\hline Range of Motion & \pm 30.00 & Degree $\left({ }^{\circ}\right)$ \\
\hline Range of Stiffness & $0 \sim \infty$ & $\mathrm{Nm} / \mathrm{rad}$ \\
\hline Maximum Torque & $\sim 3.00$ & $\mathrm{~N}^{*} \mathrm{~m}$ \\
\hline Length & 0.07 & $\mathrm{~m}$ \\
\hline
\end{tabular}

\begin{tabular}{|c|c|c|}
\hline Width & 0.08 & $\mathrm{~m}$ \\
\hline Height & 0.05 & $\mathrm{~m}$ \\
\hline Weight & 0.40 & $\mathrm{~kg}$ \\
\hline
\end{tabular}

\section{CONCLUSIONS}

In this paper, we presented the analysis and development of the CVCJ (continuously variable compliant joint), including the mechanical design, stiffness analysis and parameters identification of the novel variable stiffness joint based on the theory of the CVT-Box with variable transmission ratio. We show that when using this joint, by changing the direction of the axis of the sphere to adjust the transmission ratio we can produce a different stiffness output of the rotational joint. This results in a variable stiffness joint that can be applied across many applications. We describe the mechanical design and the structure characteristic in detail.

The novel design presented here is in its primary stages and leaves plenty opportunity for the future work. The current design results in a nonlinear relationship between stiffness and joint rotation. Future design consideration will include modifications to make the stiffness perform in a linear fashion. This will help with simpler control the transmission ratio. We also intend to build the physical model and set it to our test platform to see the real performance like the output stiffness range and the load range. According to the previous research [14], the slip of the roller with the sphere is a very important point of focus. The operating frequency of the joint, how fast it can adjust the stiffness from the lowest to the highest, is another important point that we expect to test in the future. The application is always the important part in the mechanical field so we would like to directly identify more appropriate applications for the presented joint. Ultimately, we will set the CVCJ in that robot to verify the effectiveness in a real mechanical system.

\section{References}

1. Wolf, S., Hirzinger, G., "A new variable stiffness design: Matching requirements of the next robot generation." In: IEEE International Conference on Robotics and Automation (ICRA), pp. 1741-1746. IEEE (2008)

2. Capehart T, Moore CA, Jr.. Variable Stiffness Mechanisms Using Spherical Continuously Variable Transmissions. ASME. ASME International Mechanical Engineering Congress and Exposition, Volume 4A: Dynamics, Vibration, and Control ():V04AT04A021. doi:10.1115/IMECE201551828.

3. G. A. Pratt and M. M. Williamson, "Series elastic actuators," in Intelligent Robots and Systems 95.'Human Robot Interaction and Cooperative Robots', Proceedings. 1995 IEEE/RSJ International Conference on, 1995, pp. 399-406.

4. R. A. Brooks, L. A. Stein, "Building Brains for Bodies", Autonomous Robots, vol. 1, no. 1, 1994. 
5. Van Ham R, Sugar T G, Vanderborght B, et al. Compliant actuator designs: Review of actuators with passive adjustable compliance/controllable stiffness for robotic applications[J]. IEEE Robotics and Automation Magazine, 2009, 16(3): 81-94.

6. Shane A. Migliore, E. A. Brown, and S. P. DeWeerth, Biologically inspired joint stiffness control, IEEE International Conference on Robotics and Automation, Barcelona, Spain, 2005.

7. He F B, Liang Y D, Sun J F, et al. Study on elastically actuated joints of robot for mimicking musculotendinous functions based on $\mathrm{SEAs}[\mathrm{J}]$. China Mechanical Engineering, 2014, 25(7): 900-905.

8. R. Van Ham, B. Vanderborght, M. Van Damme, B. Verrelst, and D. Lefeber, "MACCEPA, the Mechanically Adjustable Compliance and Controllable Equilibrium Position Actuator: Design and Implementation in a Biped Robot," Robotics and Autonomous Systems, vol. 55, no. 10, pp. 761-768, October 2007.

9. Rainer Bischoff, Johannes Kurth, Gunter Schreiber, Ralf Koeppe, Alin Albu-Schaffer, Alexander Beyer, Oliver Eiberger, Sami Haddadin, Andreas Stemmer, Gerhard Grunwald, and Gerhard Hirzinger. The kukadlr lightweight robot arm - a new reference platform for robotics research and manufacturing. In accepted at International Symposium on Robotics (ISR2010), 2010

10. A. Jafari, N. Tsagarakis, B. Vanderborght, and darwin Caldwell, "Awas: a novel actuator with adjustable stiffness," is in the Proceeding of IEEE/RSJ International Conference on Intelligent Robots and Systems (IROS), 2010.

11. N. G. Tsagarakis, I. Sardellitti, and D. G. Caldwell, "A new variable stiffness actuator (CompAct-VSA): design and modelling," in Proceedings of the 2011 IEEE/RSJ International Conference on Intelligent Robots and Systems: Celebrating 50 Years of Robotics
(IROS '11), pp. 378-383, IEEE, San Francisco, Calif, USA, September 2011.

12. S. Groothuis, G. Rusticelli, A. Zucchelli, S. Stramigioli, R. Carloni, "The variable stiffness actuator vsaUT-II: Mechanical design modeling and identification", IEEE/ASME Trans. Mechatronics, vol. 19, no. 2, pp. 589-597, Apr. 2014.

13. A. Jafari, N. G. Tsagarakis, I. Sardellitti, D. G. Caldwell, "A new actuator with adjustable stiffness based on a variable ratio lever mechanism", IEEE/ASME Trans. Mechatronics, vol. 19, no. 1, pp. 55-64, Feb. 2014.

14. KimS., MooreC., PeshkinM., and ColgateJ. E., 2008, "Causes of Microslip in a Continuously Variable Transmission," ASME J. Mech. Des. 130 (1), pp. 011010.

15. C. A. Moore, M. A. Peshkin, and J. E. Colgate, "Design of a 3R cobot using continuously variable transmissions," in IEEE Int. Conf. Robot. Automat., 1999

16. C. A. Moore, "Continuously variable transmission for serial link cobotarchitectures," Master's thesis, Northwestern Univ., 1997.

17. N. H. Beachley and A. A. Frank, "Continuously variable transmissions : Theory and practice," Lawrence Livermore Lab., CA, OCLC: 06690 884, 1979

18. R. B. Gillespie, C. A. Moore, M. A. Peshkin, and J. E. Colgate, "Kinematic creep in continuously variable transmissions: Traction drive mechanics for cobots," J. Mech. Syst. Design, submitted for publication.

19. FANG Li-jin, ZHOU Sheng-qi, WANG Yan. Structure Design of a New Variable Stiffness Joint. Journal of Northeastern University Nature Science, 2017, 38(12): 1748-1753. DOI: 10.12068/j.issn.10053026.2017.12.017. 